\title{
Über die Selbstvergiftung der Spirogyren im destillierten Wasser.
}

\author{
von
}

\section{Tetsu Sakamura.}

Die Beobachtung, dass das aus einer Kupferblase destillierte Wasser auf Spirogyren giftig wirkt, ist schon von NäGELI ('93) gemacht worden. Diese von ihm ,Oligodynamie“ genannte Erscheinung ist, wie er damals erkannte, auf den Gehalt an Kupfer im destillierten Wasser zurückzuführen.

Heutzutage kann man aber nicht mehr die im destillierten Wasser enthaltenen Kupfer- oder anderen șchädlichen Schwermetallionen als die einzige Ursache der Schädigung der pllanzlichen sowie tierischen Zellen betrachten, weil seit jener Zeit die Destillationsmethoden für biologische $Z$ wecke viel verbessert worden sind, wodurch Wasser erhalten wird, das von Schwermetallionen frei ist. ${ }^{\text {) }}$

In meinen Versuchen über die Einwirkung verschiedener Elektrolyte auf das Protoplasma der Spirogyren stellte sich nicht selten der Umstand ein, dass die Kontrollpflanzen in dem mit grösster Sorgfalt umdestillierten Wasser nach einigen Stunden geschädigt wurden, und schon nach Verlauf einer Nacht starben.

Diese unangenehme Erscheinung veranlasste mich, die Hauptursache dieses Absterbens der Spirogyren im destillierten Wasser aufzuklären, weil der Kontrollversuch mit destilliertem Wasser bei meinen spätern Experimenten unbedingt notwendig ist.

\section{Destillation des Wassers.}

Aus den Untersuchungen von Livingston ('07) und Hoxt ('13) geht hervor, dass die Giftstoffe im destillierten Wasser durch die Adsorptionskraft ungelöster suspendierter Substanzen, wie reiner Tier-

1) Betreffs der diesbezüglichen Literatur verweise ich auf die Arbeiten von Livingston ('07), True ('14) und Hibbard ('15). 
kohle, Eisenhydroxyd u.a., beseitigt werden können. Ich habe selbst durch Behandlung mit Merkscher Tierkohle, Verbandwatte und aschefreiem Filtrierpapier eine deutliche Verbesserung des destillierten Wasssrs erreicht. Wenn auch die Spirogyren im so behandelten destillierten Wasser unbeschädigt bleiben, so sollte man dennoch solches Wasser nicht ohne weiteres als rein betrachten und zu den Versuchen brauchen. Ich habe deshalb auch diese Behandlung des Wassers nur als einen der Destillation voranzugehenden Prozess angesehen.

Dem auf gewöhulichem Wege destillierten Wasser wurde eine mässige Menge Merkscher Tierkohle zugesetzt, und die Flasche wiederholt lebhaft geschüttelt. Ungefähr nach 20 Stunden wurde das Wasser filtriert und ihm eine kleine Menge Kaliumpermanganatlösung zugesetzt, worauf dann die Umdestillation vorgenommen wurde. Der Destillationsapparat besteht aus einer $1000 \mathrm{ccm}$. fassenden Glasretorte und einem grossräumigen kugeligen Glaskühler, damit möglichst wenig Dampf mit der Glasoberfläche in direkte Berührung kommt. ${ }^{1)}$

Die spezifische Leitfähigkeit des von mir sorgfältig umdestillierten und $\mathrm{CO}_{z}$ frei gemachten $\mathrm{W}$ assers beträgt bei $22^{\circ} \mathrm{C} .1 .9 \times 10^{-6}-\mathbf{1 , 5 5} \times 10^{-6}$ Um die H-Ionenkonzentration des Wassers zu bestimmen, wurde sowohl die Indikatoren- als auch die Gaskettenmethode verwendet. Er beträgt $\left.\mathrm{pH}=6,91-6,98^{2}\right)$ bei $22^{\circ} \mathrm{C}$. Bei den folgenden Versuchen wurde das Wasser zum Gebrauch nicht besonders kohlensäurefrei gemacht. Dem Kohlensäuregas in der Luft wurde vielmehr die Freiheit gelassen, sich entsprechend seinem Partialdruck ins Wasser zu lösen.

\section{Yersuchspfianzen.}

Im Teiche des Botanischen Gartens der hiesigen Universität gedeihen wenigstens fünf Arten von Spirogyren. In der vorliegenden Arbeit wurde nur eine allerdings mir unbekannten Artnamens, als Versuchspflanze gebraucht. Die morphologischen Eigenschaften der vegetativen Zellen sollen hier kurz beschrieben werden.

Vegetative Zellen $68 \mu$ dick, 2 mal so lang, init einfachen Scheidewänden, 1 Chromatophor mit 3-7 (gewöhnlich 6) Umgängen.

Der osmotische Druck der Zellen wurde mit Hülfe der Plasmolyse bestimmt und beträgt ca. 7,168 Atmosphären.

1) In den vorliegenden Versuchen wurde nur Normalglasware gebraucht, um das Auslösen der alkalischen Substanzen aus dem Glas ins Wasser zu vermeiden.

2) durch die Gaskettenmethode bestimmt. 


\section{Deformation des Protoplasmas der geschädigten oder sterbenden Spirogyren.}

Es ist hier nicht meine Aufgabe, die morphologischen Veränderungen des Protoplasmas bei der Schädigung ausführlich zu erörtern. Um aber in den folgenden Zeilen den Schädigungsgrad der Spirogyren in einfacher Weise bezeichnen zu können, ist nötig, kurz darauf einzugehen.

Dass das Protoplasma beim Absterben zuletzt koaguliert, ist heute eine bekannte Tatsache. Während unsere Erkenntnis der leblosen Kolloide in bezug auf Koagulation (Entwässerung, Fällung) bereits bedeutende Fortschritte gemacht hat, sind wir über das Wesen der gegenteiligen Erscheinung, nämlich der Quellung, weit weniger gut unterrichtet.

NÄGELI hat schon darauf aufmerksam gemacht, dass das Todesphänomen der Spirogyren, wenn die Pflanzen durch konzentrierte Lösungen der Schwermetallsalze geschädigt werden, sehr verschieden ist von demjenigen, das durch die verdünnten Lösungen derselben Salze verursacht wird. Er hat jenes die chemische und dieses die oligodynamische Erscheinung genannt. Wer den nähern Verlauf der Veränderung des Protoplasmas der Spirogyren im destillierten Wasser oder in der verdünnten Salzlösung verfolgt, kann ohne Schwierigkeiten erkennen, dass die Deformation mit der Quellung des Plasmas beginnt. ${ }^{\text {) }}$ Zunächst wird die Plasmaviskosität durch die Quellung erniedrigt, das Cytoplasma erhebt sich stark an der Stelle, wo die Chlorophyllbänder anhaften, und die letzteren zeigen das Bestreben, an diesen schwachen Punkten sich vom ersteren zu trennen. Bald nachher lösen sich die Chlorophyllbänder vom Plasmaschlauch ab, was das Kennzeichen des ersten Stadiums der Schädigung bedeutet. Das Cytoplasma quillt weiter nach dem Innern des Zellraumes, bis im extremen Falle der ganze Zellraum vom gequollenen Plasma erfüllt ist. Die vor kurzem von Prof. FujI ('20, '21) beschriebenen und von ihm „Ellipsonen“ genannten Körperchen treten immer mehr im Plasma auf and zeigen die Brownsche Bewegung. ${ }^{2)}$ Es wäre nicht unmöglich anzunehmen, dass die äussere Schicht des Cytoplasmas (Hautschicht ?) ursprünglich von diesen Ellipsonen durch eine Verdichtung entstanden ist, und

1) Auf die Erscheinung der direkten Koagulation des Protoplasmas möchte ich hier nicht eingehen.

2) Ich habe mit Hülfe der Dunkelfeldbeleuchtung zwei lichtbrechende Zentren in den Ellipsonen bestätigt, die zuerst von Prof. FUJIr entdeckt worden sind. 
dass ihr Zusammenhang nun durch die Quellung des Plasmas gelockert wird, wodurch sie schwimmend im Cytoplasma sich zerstreuen. Mit der Quellung des Cytoplasmas geht in den meisten Fällen diejenige der Chlorophyllbänder parallel.

Obwohl die oben erwähnten abnormen Veränderungen des Cytoplasmas je nach den Umständen in verschiedenen Graden zum Vorschein kommen, so enden sie schliesslich gleichfalls mit der Koagulation.

In den Tabellen der folgenden Versuche möchte ich der Einfachheit halber die verschiedenen Grade der Abnormitäten des Protoplasmas mit den nachstehenden Zeichen veranschaulichen:

O ............. Normal.

+ ............ Frühes Stadium der Schädigung. Das Cytoplasma erhebt sich oder die Chlorophyllbänder trennen sich davon ab.

H ........... Das, Cytoplasma sowie der Chloroplast quellen noch stärker, nachdem sich der letztere vom ersteren abgetrennt hat.

H...........Eintritt des Todes, durch die Beschädigung bedingt.

Aus einer Reihe von Orientierungsversuchen ist die folgende Tatsache mir klar geworden: Die Versuchspflanzen, die am selben Tage kurz vor dem Gebrauch aus dem Teiche genommen werden, sterben meistens im destillierten Wasser spätestens am nächsten Tage, während diejenigen, die ein oder zwei Tage im Teichwasser im Zimmer stehen bleiben, im destillierten Wasser immer normal weiterleben. ${ }^{1}$ ) Dieser Unterschied der Schädigung bei den Versuchsmaterialien veranlasste mich zu vermuten, dass der Tod der Spirogyren im destillierten Wasser in erster Linie nicht durch toxische Einflüsse des destillierten Wassers verursacht wird, sondern hauptsächlich auf die Giftwirkung der ausgeschiedenen Stoffwechselprodukte der Spirogyren zurückzuführen ist. Zur Bestätigung dieser Vermutung habe ich den folgenden "Versuch angestellt. Da als Stoffwechselprodukte möglicherweise Säuren oder andere saure Substanzen ausgeschieden werden und die saure Reaktion d.h. die H-Ionen auf die Spirogyren wahrscheinlich schädlich wirken können, so habe ich mit Hülfe der Gaskettenmethode am Anfang und am Ende des Versuchs die H-Ionenkonzentration des gebrauchten destillierten Wassers bestimmt und sie mit $\mathrm{pH}$ bezeichnet.

\section{VERSUCH I.}

Versuchsmaterial :

(A), Die Versuchspflanzen wurden am Tage vor dem Versuche mit Teichwasser zusammen frisch ins Laboratorium gebracht und in einem dunkeln Raum die

1) Nach einigen Autoren ist reines destilliertes Wasser nicht giftig für die Spirogyren. Siehe: PFEFFER ('04, s. 334). 
Nacht über stehen gelassen. Zur Zeit der Untersuchung fehlen den Pflanzen die Stärkekörner, und die Chlorophyllbänder sind abgemagert. Ich möchte solche Versuchspflanzen „Hungerpflanzen“ nennen.

(B), Die Versuchspflanzen wurden am selben Tage gesammelt und untersucht. Sie konnten eine Zeitlang unter dem direkten Sonnenlicht $\mathrm{CO}_{2}$-Assimilation ausführen. Zur Zeit des Gebrauchs sind die Versuchspflanzen reich an Stärkekörnern und ihre Chlorophyllbänder sind verdickt. Ich möchte solche Versuchspflanzen ,gesättigte Pflanzen“ nennen.

Versuchsmethode: Die Spirogyren wurden mit einer gebogenen Platinöse herausgenommen. Nach Trocknung mit Filtrierpapier, wurden sie in destilliertem Wasser ausgewaschen. Nach dreimaliger Wiederholung dieser Behandlung, kamen die Versuchspflanzen mit $100 \mathrm{ccm}$. destilliertem, Versuchswasser zusammen in einen $200 \mathrm{ccm}$. großen ERLENMEYERschen Glaskolben, der dann mit einem Gummistöpsel verschlossen wurde. Die Versuchsmaterialien wurden sogleich in einen dunkeln Raum verbracht.

\begin{tabular}{|c|c|c|c|c|c|c|}
\hline \multicolumn{3}{|c|}{ Am Anfang des Versuchs } & \multicolumn{4}{|c|}{ Am Ende des Versuchs } \\
\hline Datum. & $\begin{array}{l}\text { Versuchs- } \\
\text { pflanzen. }\end{array}$ & $\left(\begin{array}{c}\mathrm{pH} \\
(\text { Temperatur })\end{array}\right.$ & $\begin{array}{c}\text { nach } \\
\text { Stunden }\end{array}$ & $\begin{array}{c}\text { makroskop. } \\
\text { Zustand }\end{array}$ & $\begin{array}{c}\text { m }^{\mathrm{i}} \text { kroskop. } \\
\text { Zustand }\end{array}$ & $\mid \frac{\mathrm{pH}}{(\text { Temperatur })}$ \\
\hline $24 / \mathrm{VII}$ & A & $5,69\left(25^{\circ}\right)$ & 24 & normal & normal & $6,13\left(25^{\circ}\right)$ \\
\hline , & $\mathrm{B}$ & $5,57\left(25^{\circ}\right)$ & 24 & abnorm & gestorb., geschäd. & $5,37\left(25^{\circ}\right)$ \\
\hline 26/VII & A & $5,55\left(25^{\circ}\right)$ & 24 & normal & normal & $6,16\left(22^{\circ}\right)$ \\
\hline " & B & $5,49\left(25^{\circ}\right)$ & 24 & abnorm & gestorb., geschäd. & $5,17\left(22^{\circ}\right)$ \\
\hline 2/VIII & $\mathbf{A}$ & $5,72\left(23^{\circ}\right)$ & 15 & normal & normal & $6,22\left(23^{\circ}\right)$ \\
\hline ” & $\mathrm{B}$ & $6,06\left(23^{\circ}\right)$ & 15 & abnorm & gestorb., geschäd. & $4,71\left(23^{\circ}\right)$ \\
\hline 3/VIII & $\mathrm{B}$ & $5,47\left(21^{\circ}\right)$ & 18 & abnorm & gestorb., geschäd. & $4,79\left(19^{\circ}\right)$ \\
\hline $2 / \mathrm{IX}$ & A & $5,47\left(21^{\circ}\right)$ & 20 & normal & normal & $5,55\left(19^{\circ}\right)$ \\
\hline$"$ & $\mathrm{~B}$ & $5,51\left(21^{\circ}\right)$ & 20 & abnorm & gestorb., geschäd. & $5,35\left(19^{\circ}\right)$ \\
\hline $3 / \mathrm{IX}$ & A & $5,45\left(25^{\circ}\right)$ & 20 & normal & normal & $6,31\left(24^{\circ}\right)$ \\
\hline$"$ & A & $5,62\left(25^{\circ}\right)$ & 20 & normal & normal & $6,34\left(24^{\circ}\right)$ \\
\hline$"$ & $\mathbf{A}$ & $5,71\left(25^{\circ}\right)$ & 20 & normal & normal & $6,29\left(24^{\circ}\right)$ \\
\hline ", & $\mathrm{B}$ & $5,43\left(25^{\circ}\right)$ & 20 & abnorm & gestorb., geschäd. & $5,36\left(24^{\circ}\right)$ \\
\hline$"$ & $\mathrm{~B}$ & $5,60\left(25^{\circ}\right)$ & 20 & abnorm & gestorb., geschäd. & $5,14\left(24^{\circ}\right.$ \\
\hline$"$ & B & $5,59\left(25^{\circ}\right)$ & 20 & abnorm & gestorb., geschäd. & $5.04\left(24^{\circ}\right)$ \\
\hline
\end{tabular}

Aus den obigen Versuchsresultaten kann man ersehen, dass die gesättigten Pflanzen im destillierten Wasser nach einmaligem Über- 
nachten immer geschädigt werden oder absterben, und dass dabei am Ende des Versuchs die H-Ionenkonzentration erhöht wird. Die Hungerpflanzen im Gegensatz dazu bleiben ganz normal und die H-Ionenkonzentration wird niedriger. Aus diesen Tatsachen geht hervor, dass diese Veränderung der Acidität von der Natur der Versuchspflanzen d.h. von ihrem Reichtum an Reservestoffen abhängig ist. Deshalb liegt es nahe anzunehmen, dass die gestättigten Pflanzen in der Nacht order im dunklen Raum ihre Reservestoffe durch Atmung etc. verbrauchen und als Stoffwechselprodukte die Säuren ausscheiden, während bei den Hungerpflanzen wegen des Mangels an Vorratstoffen die durch deren Verbrauch bedingte Ausscheidung der Stoffwechselprodukte fehlt . Aus dieser Überlegung fliesst die Vermutung, dass die Erhöhung der Acidität des destillierten Wassers vermieden werden kann, wenn man die Pflanzen die ganze Nacht hindurch beleuchtet, woduch eine fortwährende $\mathrm{CO}_{2}$-Assimilation ermöglicht wird.

\section{VERSUCH II.}

Versuchsmaterial: Gesättigte Pflanzen.

Versuchsmethode: Fast gleich derjenigen bei dem Versuch I, aber nicht sämtliche Materialien werden in einen dunklen Raum gelegt.

I. Die Pflanzen geniessen am Tage draussen starkes diffuses Licht. Vom Untergang der Sonne an bis zum nächsten Morgen werden sie mit einer elektrischen Lampe von 60 Kerzenstärken beleuchtet.

II. Die Pflanzen bleiben fortdauernd im dunklen Raum.

\begin{tabular}{|c|c|c|c|c|c|c|}
\hline \multicolumn{3}{|c|}{ Am Anfang des Versuchs } & \multicolumn{4}{|c|}{ Am Ende des Versuchs } \\
\hline Datum & Belichtung & $\underset{\text { Temperatur })}{\mathrm{pH}}$ & $\begin{array}{c}\text { nach } \\
\text { Stunden }\end{array}$ & $\begin{array}{l}\text { makroskop. } \\
\text { Zustand }\end{array}$ & $\begin{array}{l}\text { mikroskop } \\
\text { Zustand }\end{array}$ & $\underset{(\text { Temperatur })}{\mathrm{pH}}$ \\
\hline $7 / \mathrm{VIII}$ & $\begin{array}{l}\text { beleuchtet } \\
\text { beleuchtet } \\
\text { im Dunkeln } \\
\text { im Dunkeln }\end{array}$ & $\begin{array}{c}6,21\left(24^{\circ}\right) \\
" \\
" \\
"\end{array}$ & $\begin{array}{l}20 \\
20 \\
20 \\
20\end{array}$ & $\begin{array}{l}\text { normal } \\
\text { normal } \\
\text { abnorm } \\
\text { abnorm }\end{array}$ & \begin{tabular}{|c|} 
normal \\
normal \\
gestorb., geschäd. \\
gestorb., geschäd.
\end{tabular} & $\begin{array}{l}6,51\left(24^{\circ}\right) \\
6,58\left(24^{\circ}\right) \\
5,28\left(24^{\circ}\right) \\
5,11\left(24^{\circ}\right)\end{array}$ \\
\hline 9/VIII & $\begin{array}{l}\text { beleuchtet } \\
\text { beleuchtet } \\
\text { im Dunkeln } \\
\text { im Dunkeln }\end{array}$ & $\begin{array}{c}5,94\left(24^{\circ}\right) \\
" \\
" \\
"\end{array}$ & $\begin{array}{l}24 \\
24 \\
24 \\
24\end{array}$ & $\begin{array}{l}\text { normal } \\
\text { normal } \\
\text { abnorm } \\
\text { abnorm }\end{array}$ & \begin{tabular}{|c|} 
normal, + \\
normal, + \\
gestorb., geschäd. \\
gestorb., geschäd.
\end{tabular} & $\begin{array}{l}5,63\left(24^{\circ}\right) \\
5,64\left(24^{\circ}\right) \\
4,81\left(24^{\circ}\right) \\
5,08\left(24^{\circ}\right)\end{array}$ \\
\hline 10/VIII & $\begin{array}{l}\text { beleuchtet } \\
\text { beleuchtet } \\
\text { im Dunkeln } \\
\text { im Dunkeln }\end{array}$ & $\begin{array}{c}6,02\left(24^{\circ}\right) \\
" \\
" \\
"\end{array}$ & $\begin{array}{l}24 \\
24 \\
24 \\
24\end{array}$ & $\begin{array}{l}\text { normal } \\
\text { normal } \\
\text { abnorm } \\
\text { abnorm }\end{array}$ & $\begin{array}{c}\text { normal } \\
\text { normal } \\
\text { gestorb., geschäd. } \\
\text { gestorb., geschäd. }\end{array}$ & $\begin{array}{l}6,16\left(24^{\circ}\right) \\
6,12\left(24^{\circ}\right) \\
5,26\left(24^{\circ}\right) \\
5,19\left(24^{\circ}\right)\end{array}$ \\
\hline $12 / \mathrm{VIII}$ & $\begin{array}{l}\text { beleuchtet } \\
\text { beleuchtet } \\
\text { im Dunkeln } \\
\text { im Dunkeln }\end{array}$ & $\begin{array}{c}6,09\left(24^{\circ}\right) \\
" \\
" \\
"\end{array}$ & $\begin{array}{l}24 \\
24 \\
24 \\
24\end{array}$ & $\begin{array}{l}\text { normal } \\
\text { normal } \\
\text { abnorm } \\
\text { abnorm }\end{array}$ & \begin{tabular}{|c|} 
normal \\
normal \\
gestorb., geschäd. \\
gestorb., geschäd.
\end{tabular} & $\begin{array}{l}6,15\left(23^{\circ}\right) \\
6,26\left(23^{\circ}\right) \\
5,22\left(23^{\circ}\right) \\
5,41\left(23^{\circ}\right)\end{array}$ \\
\hline
\end{tabular}


Aus der Tabelle ist klar ersichtlich, dass die oben erwähnte Annahme sehr wahrscheinlich ist. Die beleuchteten Pflanzen, auch für den Fall, dass sie gesättigt sind, vermögen die Acidität nicht $z u$ erhöhen, und werden vom Tod gerettet. Was die Natur der ausgeschiedenen sauren Substanzen betrifft, so kaum kann man nun gestützt auf die bisherigen Versuchsresultate die Entscheidung treffen. Die Kohlensäure kann doch als eine der ausgeschiedenen Substanzen genannt werden. Es fragt sich nun aber ob die Spirogyren durch die Erhöhung der Acidität geschädigt werden oder ob diese erst nach dem Absterben der Pflanzen eintritt. Es ist selbstverständlich klar, dass die am Ende des Versuchs bestimmte H-Ionenkonzentration gar nicht die schädliche Grenzkonzentration bedeutet, weil ein Teil der ausgeschiedenen Kohlensäure möglicherweise bei der Behandlung entflieht. Dazu kommt noch der Umstand, dass wenigstens die Hungerpflanzen alkalische oder weniger saure Substanzen auszuscheiden vermögen. ${ }^{1)}$ Die Reaktion des Versuchswassers wird daher durch die Spirogyren immer verändert. Dass die gesättigten Pflanzen die Acidität des destillierten Wassers stark erhöhen können, bald nachdem die Pflanzen ins Wasser eingelegt werden, ist auch aus folgendem Versuche ersichtlich.

\section{VERSUCH III.}

Versuchsmaterial: Gesättigte Pflanzen.

Versuchsmethode: Fast gleich derjenigen beim Versuch I.

Beginn: Am 9. Sept., um 2 Uhr 40 nachm.

Temperatur am Anfang des Versuchs: $21^{\circ} \mathrm{C}$.

\begin{tabular}{|c|c|c|c|c|c|c|}
\hline \multirow{2}{*}{$\begin{array}{c}\text { pH am Anfang } \\
\text { des Versuchs }\left(21^{\circ}\right)\end{array}$} & \multicolumn{2}{|l|}{ nach 22 Stunden $\left(21^{\circ}\right)$} & \multicolumn{2}{|c|}{ nach 6 Stunden $\left(20^{\circ}\right)$} & \multicolumn{2}{|c|}{ nach 20 Stunden $\left(20^{\circ}\right) \mid$} \\
\cline { 2 - 6 } & $\begin{array}{c}\text { mikroskop. } \\
\text { Zustand }\end{array}$ & $\mathrm{pH}$ & $\begin{array}{c}\text { mikroskop. } \\
\text { Zustand }\end{array}$ & $\mathrm{pH}$ & $\begin{array}{c}\text { mikroskop. } \\
\text { Zustand }\end{array}$ & $\mathrm{pH}$ \\
\hline 5,31 & + & 4,83 & + & 5,44 & $+\mathrm{H}$ & 5,35 \\
\hline
\end{tabular}

Die einmal erhöhte Acidität wird nachher wieder erniedrigt. Bei $\mathrm{pH}=4,83$ beginnt die schwache Schädigung. Es ist daher vielmehr

1) Diese Ausscheidung kann man bestätigen, wenn der Unterschied zwischen den Aciditäten am Anfang und am Ende des Versuchs mit den Hungerpflanzen in Betracht gezogen wird. Ob die gesättigten Pflanzen auch solche alkalische Substanzen ausscheiden können, ist noch nicht entschieden. 
wahrscheinlich, dass die von den Spirogyren erhöhte H-Ionenkonzentration primär die Todesursache ist, aber nicht umgekehrt. Diese Auffassung kann später auch durch eine Reihe von andern Versuchen bejaht werden.

Aus den oben erwähnten Gründen lässt sich nun aber die Beziehung zwischen der Acidität und der Schädigung der Spirogyren einzig und allein durch die Messung der H-Ionenkonzentration des Versuchswassers nicht befriedigend aufklären. Zu diesem $Z$ wecke ist es wichtig. die saure Lösung der bestimmten Säure mit verschiedenen H-Ionenkonzentrationen herzustellen, um sie momentan auf die Spirogyren wirken zu lassen. Als saure Lösungen habe ich nun folgende Lösungen der Phosphorsäure hergestellt. ${ }^{1)}$

\begin{tabular}{|c|c|c|}
\hline $\mathrm{Co}_{2}$-freies dest. Wasser (c.cm.) & $\mathrm{N} / 100 \mathrm{H}_{3} \mathrm{Po}_{4}\left(\begin{array}{l}\text { Merk } \\
\text { New York }\end{array}\right)($ c.cm. $)$ & $\mathrm{pH}\left(\right.$ bei $\left.24^{\circ}\right)$ \\
\hline 100 & 0,1 & 5,62 \\
\hline 100 & 0,2 & 5,47 \\
\hline 100 & 0,3 & 5,05 \\
\hline 100 & 0,4 & 4,74 \\
\hline 100 & 0,5 & 4,62 \\
\hline 100 & 0,7 & 4,49 \\
\hline 100 & 1,0 & 4,15 \\
\hline 100 & 1,5 & 3,90 \\
\hline 100 & 2,0 & 3,80 \\
\hline 100 & 2,2 & 3,76 \\
\hline 100 & 2,5 & 3,71 \\
\hline 100 & 3,0 & 3,54 \\
\hline
\end{tabular}

\section{VERSUCH IV.}

Versuchsmaterial: Hungerpflanzen (A) und gesättigte Pflanzen (B).

Versuchsmethode: Fast gleich derjenigen beim Versuch I, nur mit dem Unterschied, daß hierbei die Reagenzgläser ohne Stöpsel gebraucht werden.

Beginn: Am 3. Aug., 1 Uhr 30 nachm.

Temperatur am Anfang des Versuchs: $22^{\circ} \mathrm{C}$.

1) Es scheint in diesem Falle sehr zweckmäßig zu sein, Pufferlösungen zu brauchen. Es läßt sich aber dabei nicht leicht vermeiden, daß andere Kationen auftreten. 


\begin{tabular}{|c|c|c|c|}
\hline \multirow{2}{*}{$\begin{array}{c}\mathrm{pH} \text { der } \mathrm{H}_{3} \mathrm{PO}_{4}- \\
\text { Lösungen }\end{array}$} & \multirow{2}{*}{ Versuchspflanzen } & \multicolumn{2}{|c|}{ mikroskopischer Zustand } \\
\hline & & nach 1 Stunde $\left(22^{\circ}\right)$ & nach 3 Stunden $\left(22^{\circ}\right)$ \\
\hline 5,62 & $\begin{array}{l}\mathrm{A} \\
\mathrm{B}\end{array}$ & $\begin{array}{l}0 \\
0\end{array}$ & $\begin{array}{l}0 \\
0\end{array}$ \\
\hline 5,05 & $\begin{array}{l}\mathrm{A} \\
\mathrm{B}\end{array}$ & $\begin{array}{l}0 \\
0\end{array}$ & $\begin{array}{l}0 \\
0\end{array}$ \\
\hline 4,74 & $\begin{array}{l}\mathrm{A} \\
\mathrm{B}\end{array}$ & $\begin{array}{l}0 \\
0\end{array}$ & $\begin{array}{l}0+H \\
0++\end{array}$ \\
\hline 4,62 & $\begin{array}{l}\mathrm{A} \\
\mathrm{B}\end{array}$ & $\begin{array}{l}0 \\
0+\end{array}$ & $\begin{array}{l}0++ \\
0++\end{array}$ \\
\hline 4,49 & $\begin{array}{l}\mathrm{A} \\
\mathrm{B}\end{array}$ & $\begin{array}{l}0+ \\
0+\end{array}$ & $\begin{array}{l}0+H \\
++\end{array}$ \\
\hline 4,15 & $\begin{array}{l}\mathrm{A} \\
\mathrm{B}\end{array}$ & $\begin{array}{l}0+ \\
0+\end{array}$ & $\begin{array}{l}0+H(x) \\
+++\end{array}$ \\
\hline 3,90 & $\begin{array}{l}\mathrm{A} \\
\mathrm{B}\end{array}$ & $\begin{array}{l}0+ \\
0+(x)^{1}\end{array}$ & $\begin{array}{l}++0 \\
++H\end{array}$ \\
\hline 3,80 & $\begin{array}{l}\mathrm{A} \\
\mathrm{B}\end{array}$ & $\begin{array}{l}0+ \\
0+(x)\end{array}$ & $+H$ \\
\hline 3,76 & $\begin{array}{l}\mathrm{A} \\
\mathrm{B}\end{array}$ & $\begin{array}{l}++0 \\
++10\end{array}$ & $++t$ \\
\hline 3,71 & $\begin{array}{l}\mathrm{A} \\
\mathrm{B}\end{array}$ & $\begin{array}{l}++0 \\
++H\end{array}$ & ++ \\
\hline 3,54 & $\begin{array}{l}\mathrm{A} \\
\mathrm{B}\end{array}$ & ++ & $H$ \\
\hline
\end{tabular}

\section{VERSUCH V.}

Versuchsmaterial: Gesättigte Pflanzen.

Versuchsmethode: Gleich derjenigen beim Versuch IV.

Beginn: Am 30. Aug., um 2 Uhr 15 nachm.

Temperatur am Anfang des Versuchs: $24^{\circ} \mathrm{C}$.

\begin{tabular}{|c|c|c|}
\hline \multirow{2}{*}{$\mathrm{pH}_{\text {Lösungen }}^{\text {der } \mathrm{H}_{3} \mathrm{PO}_{4-}}$} & \multicolumn{2}{|c|}{$\begin{array}{l}\text { mikoskopischer } \\
\text { Zustand }\end{array}$} \\
\hline & nach 1 Stunde $\left(24^{\circ}\right)$ & nach 3 Stunden $\left(22^{\circ}\right)$ \\
\hline $\begin{array}{l}4,15 \\
3,90 \\
3,80 \\
3,76 \\
3,73 \\
3,71 \\
3,54\end{array}$ & $\begin{array}{l}0+ \\
0+ \\
0++ \\
0++(x) \\
++ \\
+++ \\
++(x)\end{array}$ & $\begin{array}{l}0+H \\
0++ \\
+0+H \\
+0+H(x) \\
++ \\
++ \\
++(x)\end{array}$ \\
\hline
\end{tabular}

1) Das Zeichen $(x)$ bedeutet stärkere Schädigung als bei ber vorhergehenden niederen H-Ionenkonzentration. 
Aus den obigen Tabellen geht hervor, dass je grösser die H-Ionenkonzentration ist, desto stärker die Spirogyren geschädigt werden, und dass die giftige Grenzkonzentration der H-Ionen uugetähr $\mathrm{pH}=\mathbf{5}$ beträgt. Diese Grenzkonzentration ergibt sich auch mit der Kohlensäure im Versuch III sowie im nächsten Versuch.

\section{VERSUCH VI.}

Versuchsmaterial: Gesättigte Pflanzen.

Versuchsmethode: Gleich derjenigen beim Versuch I. In destilliertes Wasser wurde etwas Kohlensäuregas eingeblasen und die Acidität dadurch ein wenig erhöht. Die H-Ionenkonzentration des destillierten Wassers beträgt am Anfang des Versuchs $\mathrm{pH}=5,02$ bei $26^{\circ} \mathrm{C}$.

Beginn: Am 5. Sept., um 2 Uhr 30 nachm.

Temperatur am Anfang des Versuchs: $26^{\circ} \mathrm{C}$.

\begin{tabular}{|c|c|c|}
\hline $\begin{array}{c}\text { nach } \\
\text { Stunden }\end{array}$ & $\begin{array}{c}\text { mikroskopischer } \\
\text { Zustand }\end{array}$ & $\mathrm{pH}$ (Temperatur) \\
\hline $1 / 2$ & $0+$ & $5,29\left(26^{\circ}\right)$ \\
1 & $0+$ & $5,39\left(26^{\circ}\right)$ \\
2 & $0+$ & $5,34\left(26^{\circ}\right)$ \\
3 & $0+$ & $5,34\left(26^{\circ}\right)$ \\
4 & $0+$ & $5,29\left(26^{\circ}\right)$ \\
20 & $0+$ & $5,78\left(26^{\circ}\right)$ \\
\hline
\end{tabular}

Im Laufe dieses Versuchs wurde das Wasser manchmal herausgenommen, um die H-Ionenkonzentration $\mathrm{zu}$ bestimmen, und dies hat der akkumulierenden Kohlensäure Gelegenheit gegeben von Wasser zu entfliehen. Die Schädigung tritt am Anfang nur schwach auf, und zeigt selbst in einem Zeitraum von 20 Stunden keine weitere Zunahme. Von Interesse ist $z u$ sehen, dass hierbei vier Stunden lang keine merkliche Veränderung der H-Ionenkonzentration zu konstatieren ist. Dies zeigt, dass nur die schon am Anfang vorhandenen H-Ionen in der Konzentration $\mathrm{pH}=5,02$ auf die Spirogyren schwach schädlich gewirkt haben, und dass die H-Ionen in der sekundär ganz zufällig erniedrigten Konzentration keine solche Giftwirkung mehr ausüben.

Wir wollen nun unsere Aufmerksamkeit auf die osmotische Beziehung richten.

Bisher ist diese in meinen Versuchen gar nicht in Betracht gezogen worden, deshalb erhebt sich nun die Frage, ob die Hypotonie des destillierten Wassers an der schädlichen Wirkung teilnehmen kann. 
Wie schon von J. Loeb ('99-'00, '03), Osterhout '('13), True ('14) u.a. betont wurde, wäre es auch möglich, dass das destillierte Wasser dem Protoplasma die ionisierten Proteide oder anorganischen Salze aussaugt. Dass aber die Aussaugkraft in uuserem Falle keine Hauptrolle spielt, kann man leicht daran ersehen, dass selbst die gesättigten Pflanzen durch mehrmalige Erneuerung des Wassers noch einige Tage lang frisch bleiben und normal weiter leben können. Ja, bei den Hungerpflanzen kommt selbst bei längerem Verbleiben in destilliertem Wasser keine bemerkenswerte Schädigung vor.

Das Aussaugen ist eigentlich nicht als eine einfache osmotische Erscheinung zu betrachten, sondern muss als ein noch komplizierterer elektrosmotischer Prozess aufgefasst werden. ${ }^{1)}$ Unter den osmotischen Erscheinungen der Zellen möchte ich daher nur die Beziehung des Druckes zwischen dem Zellsaft im miksoskopisch sichtbaren Vakuol und der umgebenden Flüssigkeit verstehen.

Es ist erforderlich, zunächst den osmotischen Druck des Zellsaftes der Spirogyren mit demjenigen des Teichwassers, in dem sie vorkommen, zu vergleichen.

Osmotischer Druck des Teichwassers (durch Kryoskopie bestimmt) $=0,2408-0,4816$ Atmosphären.

Osmotischer Druck des Zellsaftes (durch Plasmolyse bestimmt) $=7,168$ Atmosphären.

Man kann daraus sogleich klar ersehen, wie gross der Unterschied $z$ wischen den beiden osmotischen Drücken ist. Die meisten pflanzlichen Zellen sind immer durch die Umhüllung der Zellmembran vom Zerplatzen im hypotonischer Flüssigkeit geschützt, ja die dadurch erfolgte starke Spannung (Turgeszenz) ist, ökologisch betrachtet, vielmehr eine zweckmässige Vorkehrung bei so einfach fadenförmig organisierten Organismen wie die Spirogyren, um den eigenen Körper fest zu behalten.

Die gesättigten Spirogyren wurden in Traubenzuckerlösungen von verschiedener Konzentration $1 \times 10^{-5}$ bis $5 \times 10^{-2} \mathrm{Mol}$ gelegt, und es wurde geprüft, ob auch hierbei die Spirogyren besonders in den verdünnten Lösungen stark beeinflusst werden. Das ist aber tatsächlich

1) Die Osmose kann nach VAN'T HoFF einfach thermodynamisch, d.h. durch die Gesetze des Gases erklärt werden. Das Wasser, das im Protoplasma als Quellungswasser vorhanden ist, ist aber durch elektrosmotische Adsorption (kapillare Imbibition mit elektrischer Ladung) zwischen den Teilchen der dispersen Phase der Plasmakolloide festgehalten. Die Beziehungen zwischen dieser Plasmaflüssigkeit und der umgebenden Flüssigkeit können nicht so einfach osmotisch erklärt werden. 
nicht der Fall. In allen diesen Traubenzuckerlösungen geschah die Schädigung gleichmässig ebensostark wie im destillierten Wasser. Gestützt auf diese Tatsache ist nicht daran zu zweifen, dass die starke Hypotonie des destillierten Wassers keine besondere schädliche osmotische Wirkung ausübt.

Überblicken wir die oben erwähnten Versuchsresultate, so halten wir uns zu dem Schluss berechtigt, dass die Schädigung der Spirogyren im sorgfältig destillierten Wasser hauptsächlich auf die durch die Stoffwechselprodukte erhöhte Acidität, nämlich die H-Ionenkonzentration, zurückzuführen ist.

Es wird heutzutage allgemein angenommen, dass verschiedene physiologische Wirkungen, besonders die Giftwirkungen der Säuren hauptsächlich von ihren H-Ionen abhängen, während ihre Moleküle dabei keine besondere Rolle spielen. Auch bei unseren Versuchen mit Spirogyren ist eine derartige Wirkung der H-Ionen der ausgeschiedenen Säure sehr wahrscheinlich, wenn auch das Wasen der ausgeschiedenen Substanzen noch unklar bleibt.

Bei einigen Süsswasseralgen sind aber von NAKANo ('17) etwas anders geartete Verhältnisse angegeben worden. Nach seiner Mitteilung wirken die stark dissozierbaren anorganischen Säuren, wie Salzsäure, Schwefelsäure und Salpetersäure, auf Chlorella, Stichococcus u.a. gegen unsere Erwartung schwächer als die verhältnismässig schwach dissozierbaren organischen Säuren wie Ameisensäure u.a.. Chlorella und Chlorosphaera z.B. können noch in $\frac{\mathrm{N}}{10} \mathrm{HCI}^{1)}$ und $\mathrm{H}_{2} \mathrm{SO}_{4}$ ganz frisch bleiben, während die Ameisensäure schon bei $\frac{N}{500}$ schädlich wirkt. Aus diesem überraschenden Versuchsresultat hat er geschlossen, dass die Giftwirkung dieser Säuren jedenfalls deren Molekülen zugeschrieben werden muss, nicht aber den dissozierten H-Ionen.

Nun erhebt sich die Frage, ob dieselbe Auffassung auch bei unseren Spirogyren gültig ist, d.h. ob vorzüglich die Moleküle der ausgeschiedenen Säuren als Wirkungsfaktoren betrachtet werden müssen. Um die Frage zu entscheiden, habe ich die folgenden Versuche angestellt, bei denen ich zwei Reihen von Reaktionsregulatoren anwendete ${ }^{2)}$ :

1) Die H-Ionenkonzentration dieser Lösung beträgt $[\mathrm{H} \cdot]=0,084, \mathrm{pH}=1,071$ bei $18^{\circ} \mathrm{C}$.

2) Über die ausführliche Theorie und Vorschrift der Regulatoren siehe: Michaelis ('14) s. 182. 


\begin{tabular}{|c|c|c|c|}
\hline & (1) & (2) & (3) \\
\hline$\frac{\mathrm{N}}{10} \mathrm{CH}_{3} \mathrm{COONa}(\mathrm{cm})$. & 1 & 1 & 1 \\
\hline$\frac{\mathrm{N}}{10} \mathrm{CH}_{3} \mathrm{COOH}$ (ccm.) & 0,64 & 1,28 & 2,56 \\
\hline $\begin{array}{l}\mathrm{CO}_{2} \text {-freies dest. } \\
\text { Wasser (ccm.) }\end{array}$ & 8,36 & $17,72(7,72)$ & $36,44(6,44)$ \\
\hline $\begin{array}{l}\text { Konzentration } \\
\text { der Essigsäure }\end{array}$ & $\frac{6,4}{1000} \mathrm{~N}$ & $\frac{6,4}{1000} N\left(\frac{12,8}{1000} N\right)$ & $\frac{6,4}{1000} \mathrm{~N}\left(\frac{25,6}{1000} \mathrm{~N}\right)$ \\
\hline $\mathrm{pH}$ & 4,89 & 4,59 & 4,28 \\
\hline & (4) & (5) & (6) \\
\hline$\frac{\mathrm{N}}{10} \mathrm{CH}_{3} \mathrm{COO} \mathrm{Na}(\mathrm{ccm})$. & 1 & 1 & 1 \\
\hline$\frac{\mathrm{N}}{100} \mathrm{CH}_{3} \mathrm{COOH}$ (ccm.) & 3,2 & & . \\
\hline$\frac{\mathrm{N}}{100} \mathrm{CH}_{3} \mathrm{COOH}$ (ccm.) & & 0,64 & 1,28 \\
\hline $\begin{array}{l}\mathrm{CO}_{2} \text {-freies dest. } \\
\text { Wasser (ccm.) }\end{array}$ & 5,8 & $18,36(8,36)$ & $37,72(7,72)$ \\
\hline $\begin{array}{l}\text { Konzentration } \\
\text { der Essigsäure }\end{array}$ & $\frac{3,2}{1000} \mathrm{~N}$ & $\frac{3,2}{1000} \mathrm{~N}\left(\frac{6,4}{1000} \mathrm{~N}\right)$ & $\frac{3,2}{1000} \mathrm{~N}\left(\frac{1,28}{1000} \mathrm{~N}\right)$ \\
\hline $\mathrm{pH}$ & 5,17 & 4,89 & 4,59 \\
\hline
\end{tabular}

Verglichen mit Michaelis' Vorschrift ('14, s. 184) ist bei meinen Lösungen (2), (5) und (3), (6) die Verdünnung mit Wasser zweimal bzw. viermal geringer, ${ }^{1)}$ Dass die H-Ionenkonzentration eines Regulators nicht merklich verändert wird, trotzdem die Lösung mit Wasser verdünnt wird, kann man mit Hülfe der Indikatorenmethode ohne Schwierigkeiten beweisen. Die H-Ionenkonzentration bei unseren Acetatgemischen hängen also nur von ,dem Verhältnis von freier Essigsäure zum Natriumacetat ab. ${ }^{2)}$

Nun haben wir also Pufferlösungen, deren H-Ionenkonzentrationen voneinander verschieden sind, während die Konzentrationen der gegebenen freien Sätre gleich sind.

\section{VERSUCH VII.}

Versuchsmaterial: Hungerpflanzen.

Versuchsmethode: Fast gleich derjenigen beim Versuch I, nur mit dem Unterschied, daß Reagenzgläser statt ERLENMEYERscher Kolben gebraucht werden.

Beginn : Am 8, Sept., um 2 Uhr nachm.

Temperatur am Anfang des Versuchs: $21^{\circ} \mathrm{C}$.

1) Die eingeklammerten Zahlen in den Tabellen sind von Mrchatels gegeben.

2) Michatis ('21) s. 22-23. 


\begin{tabular}{|c|c|c|}
\hline \multirow{2}{*}{ Pufferlösung ( $\mathrm{pH}$ ) } & \multicolumn{2}{|c|}{ mikroskopischer Zustand } \\
\hline & nach 1 Stunde $\left(21^{\circ}\right)$ & nach 2 Stunden $\left(21^{\circ}\right)$ \\
\hline $\begin{array}{l}\text { (1) } 4,89 \\
\text { (2) } 4,59 \\
\text { (3) } 4,28\end{array}$ & $\begin{array}{l}0+H \\
+0 H \\
+H\end{array}$ & $\begin{array}{l}+0 H \\
H \\
H\end{array}$ \\
\hline $\begin{array}{l}\text { (4) } 5,17 \\
\text { (5) } 4,89 \\
\text { (6) } 4,59\end{array}$ & $\begin{array}{l}0 \\
0+ \\
+0+\end{array}$ & $\begin{array}{l}0+\text { (sehr wenig) } \\
+0+H \\
+H\end{array}$ \\
\hline
\end{tabular}

Aus dieser Tabelle ist es ersichtlich, dass die schädliche Wirkung auf die Spirogyren entsprechend der steigenden H-Ionenkonzentration zunimmt, obschon die Konzentration der verwendeten Essigsäure dabei gleich bleibt.

Wenn auch J. Loeb ('09) und CzapeK ('11) bereits darauf aufmerksam gemacht haben, dass die Fettsäuren viel stärkere physiologische Wirkungen entfalten, als man nach ihrem Dissoziationsgrade erwarten sollte, so wäre es nicht richtig, in unserem Falle die Giftwirkung in erster Linie den Molekülen der Säure zuzuschreiben. Man muss dabei erkennen, dass diese verschiedenen Schädigungsgrade hauptsächlich von den Unterschieden der H-Ionenkonzentrationen der dissozierten Essigsäure abhängig sind. Natürlich ist es möglich, dieselbe Deutung auch auf andere Säuren auszudehnen, und man braucht nicht auf den schon geäusserten Schluss zu verzichten.

\section{Schutz der Spirogyren vor Selbstrergiftung im destillierten Wasser.}

Insofern die Selbstvergiftung der Spirogyren durch die Erhöhung der H-Ionenkonzentration im destillierten Wasser verursacht wird und das Vorhandensein von Kohlensäure als einem der ausgeschiedenen Stoffwechselprodukte wahrscheinlich ist, vermag man die Spirogyren theoretisch durch Neutralisation oder durch Anwendung von Carbonaten oder Bicarbonaten zwecks Pufferwirkung zu schützen.

Zunächst möchte ich hier das Resultat des Neutralisationsversuchs mitteilen. 


\section{VERSUCH VIII.}

Versuchsmaterial : Gesättigte Pflanzen.

Versuchsmethode: Gleich derjenigen beim Versuch I

$\frac{\mathrm{N}}{1000} \mathrm{NaOH}$ (Merk)-Lösung wurde hergestellt. Ihr pH-Wert beträgt 11,93 be $21^{\circ} \mathrm{C}$, theoretisch aber 12,13 bei $18^{\circ} \mathrm{C}$.

Beginn: Am 7. Sept., um 2 Uhr nachm.

Temperatur am Anfang des Versuchs: $21^{\circ} \mathrm{C}$.

\begin{tabular}{|c|c|}
\hline $\begin{array}{c}\text { nach } \\
\text { Stunden (Temperatur) }\end{array}$ & mikroskopischer Zustand. \\
\hline $11 / 2\left(21^{\circ}\right)$ & normal \\
\hline $24\left(21^{\circ}\right)$ & normal, Anordnung der Chlorophyllbänder etwas abnorm. \\
\hline $48\left(21^{\circ}\right)$ & $"$ \\
\hline $72\left(21^{\circ}\right)$ & $\begin{array}{l}\text { normal, Chlorophyllbänder sehr dünn, Zellen teilweise } \\
\text { gestorben. }\end{array}$ \\
\hline $96\left(21^{\circ}\right)$ & $\begin{array}{l}\text { fast normal, Chlorophyllbänder sehr dünn und etwas } \\
\text { verlängert. Zellen teilweise gestorben. }\end{array}$ \\
\hline
\end{tabular}

Dieses Versuchsresultat zeigt uns, dass $\mathrm{NaOH}$ durch seine Neutralisationskraft bis zu einem gewissen Grade die Giftwirkung der ausgeschiedenen sauren Substanzen auszuschalten vermag. Die Spirogyren ertragen daher eine ziemlich hohe Alkalität; denn alkalische Medien sind ihrem Gedeihen günstiger als saure. Beirn Versuche mit $\mathrm{NaOH}$ lässt sich aber die Wirkung der gleichzeitig vorkommenden Na-Ionen nicht vermeiden, deren schädlichem Einfluss wohl die oben erwähnten schwachen Abnormitäten der Chlorophyllbänder zur Last gelegt werden können.

Im Gemisch einer schwachen Säure mit einem ihrer Salze wird die Dissoziation der ersteren stark herabgesetzt, wobei die H-Ionenkonzentration fast ausschliesslich von dem Verhältnis der Säure zum Salze abhängig ist. Gestützt auf diese Erwägung habe ich eine Reihe von Versuchen mit Na-Bicarbonat und Ca-Carbonat ${ }^{1)}$ angestellt, um zu prüfen, ob sie eine Schutzwirkung gegen die schädliche Acidität besonders der Kohlensäure ausüben können.

VERSUCH IX. a.

Versuchsmaterial: Gesättigte Pflanzen.

Versuchsmethode: Glleich derjenigen beim Versuch I.

Beginn: Am 17. Aug., um 5 Uhr 30 nachm.

Temperatur am Anfang des Versuchs: $27^{\circ} \mathrm{C}$.

1) Ca-Carbonat verbindet sich mit Kohlensäure und daraus wird Ca-Bicarbonat erzeugt. 
VERSUCH IX. $b$.

Versuchsmaterial: Gesättigte Pflanzen.

Versuchsmethode: Gleich derjenigen beim Versuchs I.

Beginn; Am 24. Aug., um 2 Uhr 30 nachm.

Temperatur am Anfang des Versuchs: $25^{\circ} \mathrm{C}$.

\section{(a.)}

\begin{tabular}{|c|c|c|c|c|c|}
\hline \multirow{2}{*}{$\begin{array}{c}\text { molare } \\
\text { Konzent. der } \\
\mathrm{NaHCO}_{\mathbf{3}^{-}} \\
\text {Lösung. }^{-}\end{array}$} & \multicolumn{2}{|c|}{ nach 24 Stunden $\left(28^{\circ}\right)$} & \multicolumn{3}{|c|}{ nach 43 Stunden $\left(27^{\circ}\right)$} \\
\hline & $\begin{array}{c}\text { makroskop. } \\
\text { Zustand }\end{array}$ & $\begin{array}{c}\text { mikroskop. } \\
\text { Zustand }\end{array}$ & $\begin{array}{c}\text { makroskop. } \\
\text { Zustand }\end{array}$ & $\begin{array}{c}\text { mikroskop. } \\
\text { Zustand }\end{array}$ & $\mathrm{pH}$ \\
\hline $1 \times 10^{-5}$ & abnorm & $H$ & abnorm & HH & 6,04 \\
\hline $5 \times 10^{-5}$ & abnorm & $\mathrm{H}$ & abnorm & HH & 6,18 \\
\hline $1 \times 10^{-4}$ & abnorm & $\mathrm{HH}$ & abnorm & $\mathrm{HH}$ & 6,18 \\
\hline $5 \times 10^{-4}$ & etwas abnorm & $0+H$ & abnorm & $+H+H$ & 6,47 \\
\hline $1 \times 10^{-3}$ & etwas abnorm & $0+$ & etwas abnorm & $+0 \mathrm{H}$ & 6,68 \\
\hline $5 \times 10^{-3}$ & normal & 0 & fast normal & $0+H$ & 7,24 \\
\hline $1 \times 10^{-2}$ & norn & & normal & & 7,88 \\
\hline $5 \times 10^{-2}$ & anscheinend normal & $+H+H \triangle^{1}$ & anscheinend normal & $+H+H \Delta$ & 8,41 \\
\hline $1 \times 10^{-1}$ & anscheinend normal & $+H+H \Delta$ & anscheinend normal & $+H+H \Delta$ & 8,41 \\
\hline dest. Wasser. & abnorm & H & abnorm & $\mathrm{HH}$ & 5,16 \\
\hline
\end{tabular}

(b.)

\begin{tabular}{|c|c|l|l|l|}
\hline \multicolumn{2}{|c|}{$\mathrm{NaHCO}_{3}$} & \multicolumn{3}{|c|}{ nach 24 Stunden $\left(23^{\circ}\right)$} \\
\cline { 1 - 4 } Mol. & $\mathrm{pH}$ & $\begin{array}{c}\text { makroskop. } \\
\text { Zustand }\end{array}$ & $\begin{array}{c}\text { mikroskop. } \\
\text { Zustand }\end{array}$ & $\mathrm{pH}$ \\
\hline $1 \times 10^{-5}$ & 6,38 & abnorm & $+0+\mathrm{H}$ & 5,37 \\
$1 \times 10^{-4}$ & 6,67 & abnorm & $0+\mathrm{H}$ & 5,64 \\
$1 \times 10^{-3}$ & 7,91 & etwas abnorm. & $0+$ & 6,51 \\
$1 \times 10^{-2}$ & 8,70 & normal & 0 & 7,82 \\
$1 \times 10^{-1}$ & 8,80 & anscheinend normal & ++++ + & 8,47 \\
dest. Wasser. & 5,94 & abnorm & $+0+\mathrm{H}$ & 5,15 \\
\hline
\end{tabular}

Wie aus den Tabellen ersichtlich ist, besitzt Na-Bicarbonat nur in der Konzentration $1 \times 10^{-2} \mathrm{Mol}$ ein Schutzvermögen für die Spirogyren. Bei niedrigern Konzentration sind die Lösungen nicht mehr imstande, die Dissoziation der Säure so stark herabzusetzen, dass die Spirogyren

1) Die Zellen sind reich an Stärkekörnẹn 
nicht geschädigt werden. Anderseits büssen die konzentrierten Lösungen ihre Schutzfähigkeit deshalb ein, weil die ihnen innewohnenden Na-Ionen eine schädliche Wirkung ausüben. Dieser Nachteil kann aber durch Gebrauch von Ca-Carbonat statt Na-Bicarbonat beseitigt werden.

Ca-Ionen besitzen ein eigentliches Vermögen, das Protoplasma in festem Zustande $z \overline{\mathbf{u}}$ erhalten und gegen die für die Zellen schädlichen Kationen antagonistisch zu wirken. ${ }^{1)}$ Soweit meine Kenntnis der Literatur reicht, ist es aber wenig bekannt, dass sie dieselbe antagonistische Wirkung auch gegen die H-Ionen entfalten können.

Es ist unbedingt erforderlich, zunächst die antagonistische Wirkung der Ca-Ionen von $\mathrm{CaCl}_{2}$ gegen die $\mathrm{H}$-Ionen zu bestätigen, bevor Ca-Carbonat als Schutzmittel für die Spirogyren geprüft wird. $\mathrm{KCl}$ diente als Kriterium, um zu untersuchen, ob auch die K-Ionen gegen die H-Ionen dieselbe antagonistische Wirkung besitzen können.

\section{VERSUCH X. a.}

Versuchsmaterial: Gesättigte Pflanzen.

Versuchsmethode: Gleich derjenigen beim Versuch $I$.

Folgende drei Flüssigkeiten wurden zum Gebrauch hergestellt:

(1) Destilliertes Wasser.

(2) $\frac{\mathrm{N}}{10} \mathrm{CaCl}_{2}$ (MERK)

(3) $\frac{\mathrm{N}}{10} \mathrm{KCl}$ (MERK)

Bei allen diesen Flüssigkeiten war der ungeführe Wert von $\mathrm{pH}=5,32$ bei $20^{\circ} \mathrm{C}$.2) Beginn: Am 6. Sept., Mittag.

Temperatur am Anfang des Versuchs: $20^{\circ} \mathrm{C}$.

\section{Versuch X. $b$.}

Versuchsmaterial: Gesättigte Pflanzen.

Versuchsmethode: Gleich derjenigen beim Versuch I.

Folgende zwei Lösungen wurden zum Gebrauch hergestellt:

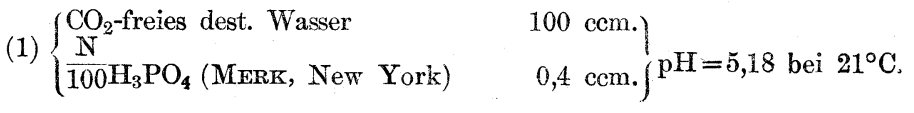

1) Die Verhinderung der Keimung oder des Wachstums der Samen oder Sporen durch (a-Ionen deute ich nicht als schädliche Wirkung.

2) In die Lösung (2) wurde etwas $\mathrm{CO}_{2}$ eingeblasen, um die Acidität der drei Flüssigkeiten einheitlich zu machen. 
(2) $\left\{\begin{array}{ll}\frac{\mathrm{N}}{10} \mathrm{CaCl}_{2}(\mathrm{MeRK}) & 100 \mathrm{ccm} . \\ \frac{\mathrm{N}}{100} \mathrm{H}_{3} \mathrm{PO}_{4}(\text { MerK, New York }) & 0,8 \mathrm{ccm} .\end{array}\right\} \mathrm{pH}=4,87$ bei $21^{\circ} \mathrm{C}$.

Beginn: Am 7. Sept., um 4 Uhr nachm.

Temperatur am Anfang des Versuchs: $21^{\circ} \mathrm{C}$.

(a.)

\begin{tabular}{|c|c|c|c|c|c|c|c|c|c|}
\hline \multirow{2}{*}{ 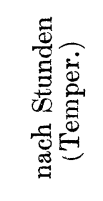 } & \multicolumn{3}{|c|}{ dest. Wasser } & \multicolumn{3}{|c|}{$\mathrm{CaCl}_{2}$} & \multicolumn{3}{|c|}{$\mathrm{KCl}$. } \\
\hline & 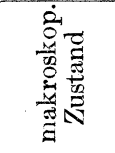 & 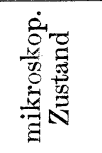 & $\mathrm{pH}$ & 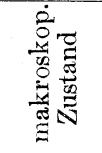 & 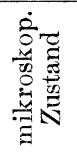 & $\mathrm{pH}$ & 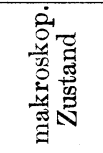 & 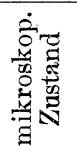 & $\mathrm{pH}$ \\
\hline $5\left(21^{\circ}\right)$ & normal & 0 & - & normal & 0 & - & normal & $H$ & 一 \\
\hline $20\left(21^{\circ}\right)$ & abnorm & $0+$ & - & abnorm & 0 & - & abnorm & $H$ & - \\
\hline $29\left(21^{\circ}\right)$ & abnorm & $0 \mathrm{HH}$ & 5,81 & abnorm & 0 & 5,86 & abnorm & $H$ & - \\
\hline $50\left(21^{\circ}\right)$ & abnorm & $0 \mathrm{HH}$ & 5,83 & abnorm & 0 & 5,88 & abnorm & $H$ & 一 \\
\hline
\end{tabular}

(b.)

\begin{tabular}{|c|c|c|c|c|c|c|}
\hline \multirow{2}{*}{$\begin{array}{c}\text { nach } \\
\text { Stunden (Temper.) }\end{array}$} & \multicolumn{3}{|c|}{ (1) } & \multicolumn{3}{|c|}{$(2)^{\circ}$} \\
\hline & $\begin{array}{c}\text { makroskop. } \\
\text { Zustand }\end{array}$ & $\begin{array}{l}\text { mikroskop. } \\
\text { Zustand }\end{array}$ & $\mathrm{pH}$ & $\begin{array}{l}\text { makroskop. } \\
\text { Zustand }\end{array}$ & $\begin{array}{l}\text { mikroskop. } \\
\text { Zustand }\end{array}$ & $\mathrm{pH}$ \\
\hline $11 / 2\left(21^{\circ}\right)$ & normaī & 0 & - & normal & 0 & - \\
\hline $24 \quad\left(21^{\circ}\right)$ & abnorm & $+H$ & 4,87 & abnorm & 0 & 4,92 \\
\hline $48 \quad\left(21^{\circ}\right)$ & abnorm & $H$ & 5,47 & abnorm & $\begin{array}{c}\left.0^{1}\right) \\
\end{array}$ & 5,73 \\
\hline $72 \quad\left(21^{\circ}\right)$ & abnorm & $H$ & - & abnorm & $\begin{array}{r}+(\operatorname{sen} r \text { wenig) } \\
01) \\
+(\operatorname{sehr} \text { wenig) }\end{array}$ & 5,85 \\
\hline
\end{tabular}

Die Ca-Ionen sind deutlich gegen die H-Ionen antagonistisch wirksam, während die K-Ionen noch additiv schädlich auf die Spirogyren wirken. Aus dem Versuch $\mathrm{X}$ b kann man ersehen, dass die Spirogyren nach 24 Stunden $\left(21^{\circ} \mathrm{C}\right)$ in der Lösung (2) mit Ca-Ionen noch bei $\mathrm{pH}=4,92$ unter dem Mikroskope ganz normal erscheinen, obschon ihr makroskopischer Zustand abnorm ist. In der Lösung (1), der die Ca-Ionen fehlen, werden die Spirogyren ohne Schutz so stark geschädigt, wie im destillierten Wasser mit derselben H-Ionenkonzentration.

1) Die Chlorophyllbänder sind dünn. 
Aus den bisherigen Versuchen und Erwägungen dürfen wir nun erwarten, dasz dem Ca-Carbonat zweierlei Vermögen, eine Puffer- und eine antagonistische Wirkung gegen die H-Ionen zukommt. Der folgende Versuch mit Ca-Carbonat zeigt uns eine interessante Tatsache, die zur Bestätigung unserer Erwartung dienen kann.

\section{VERSUCH XI.}

Versuchsmaterial: Gesättigte Pflanzen.

Versuchsmethode: Fast gleich derjenigen beim Versuch I, mit dem Unterschied, daB die Pflanzen in ERLenneyersche Kolben unter diffuses Tageslicht gesetzt werden.

Beginn: Am 6. Sept., um 2 Unr 30 nachm.

Temperatur am Anfang des Versuchs: $21^{\circ} \mathrm{C}$.

\begin{tabular}{|c|c|c|c|c|c|c|}
\hline \multirow[b]{2}{*}{$\begin{array}{l}\text { nach Tagen } \\
\text { (Temper.) }\end{array}$} & \multicolumn{6}{|c|}{ Makroskopischer Zustand. } \\
\hline & 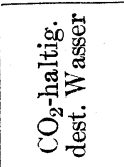 & 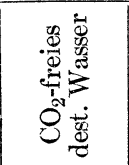 & 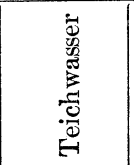 & 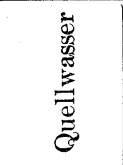 & $\begin{array}{r}\text { dest. Wasser mit } \\
\text { gesättigt.CaCO }{ }^{1} \text { ) }\end{array}$ & $\begin{array}{l}\text { dest. Wasser mit } \\
\text { überschüssig. } \\
\left.\mathrm{CaCO}_{3}^{2}\right)\end{array}$ \\
\hline $1\left(21^{\circ}\right)$ & fast normal & fast normal & normal & normal & normal & - normal \\
\hline $2\left(21^{\circ}\right)$ & abnorm & etwas abnorm & normal & normal & normal & normal \\
\hline $3\left(21^{\circ}\right)$ & abnorm & abnorm & normal & normal & normal & normal \\
\hline $4\left(21^{\circ}\right)$ & abnorm & abnorm & fast normal & normal & normal & normal \\
\hline $5\left(21^{\circ}\right)$ & abnorm & abnorm & etwasabnorm & fast normal & normal & normal \\
\hline $6\left(21^{\circ}\right)$ & abnorm & abnorm & etwas abnorm & fast normal & normal & normal \\
\hline
\end{tabular}

\begin{tabular}{|c|c|c|c|c|c|c|}
\hline \multirow[b]{2}{*}{$\begin{array}{c}\text { nach Tagen } \\
\text { (Temper.) }\end{array}$} & \multicolumn{6}{|c|}{ Makroskopischer Zustand. } \\
\hline & 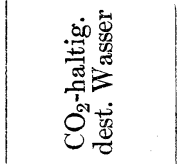 & 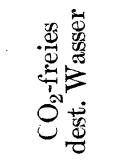 & 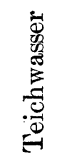 & 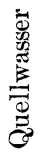 & $\begin{array}{c}\text { dest. Wasser mit } \\
\text { gesättigt.CaCO }{ }_{3}^{3} \text { ) }\end{array}$ & $\begin{array}{l}\text { dest. Wasser mit } \\
\text { überschüssig. } \\
\left.\mathrm{CaCO}_{3}^{4}\right)\end{array}$ \\
\hline $1\left(21^{\circ}\right)$ & $0+$ & 0 & 0 & 0 & 0 & 0 \\
\hline $2\left(21^{\circ}\right)$ & $0+H+H$ & 0 & 0 & 0 & 0 & 0 \\
\hline $3\left(21^{\circ}\right)$ & $0++H$ & $0++$ & 0 . & 0 & 0 & 0 \\
\hline $4\left(21^{\circ}\right)$ & $\left.\times \times x^{3}\right)$ & $\times \times$ & $\times$ & 0 & 0 & 0 \\
\hline $5\left(21^{\circ}\right)$ & $x \times x$ & $x \times$ & $\times x$ & $\times$ & 0 & 0 \\
\hline $6\left(21^{\circ}\right)$ & $\times \times \times$ & $x \times$ & $x \times$ & $x$ & $x$ & $0^{4)}$ \\
\hline
\end{tabular}

1) Calciumcarbonat im Überschuß wurde dem destillierten Wasser zugesetzt und die Lösung nachher filtriert.

2) Calciumcarbonat im Überschuß wurde dem destillierten Wasser zugesetzt.

3) Das Zeichen $(x)$ bedeutet den Schädigunsgrad durch die Infektion mit Lagenidium Rabentorstii (?).

4) In der Lösung können die Spirogyren noch ungeschädigt weiter gedeihen. 
Man sieht aus diesen Tabellen, dass es ein charakterisches Vermögen von $\mathrm{CaCO}_{3}$ ist, die Spirogyren vor der Selbstvergiftung zu schützen, obwohl der Versuch durch die Infektion mit Lagenidium(?) etwas gestört wurde. ${ }^{1)}$

In der Natur kommt Calciumcarbonat verhältnismäszig in grosser Menge im Teich-, Quell-, und Bachwasser vor, in dem die Spirogyren gedeihen, was als sehr zweckmässig betrachtet werden muss. Diese interessante Tatsache erinnert im grossen und ganzen an die Verhältnisse im Blut, worin das Bicarbonat und das Phosphat die Reaktion regulieren. ${ }^{2)}$ Es ist daher sehr empfehlenswert bei der Kultur der Spirogyren eine überschüssige Menge von $\mathrm{CaCO}_{3}$ dem Wasser zuzusetzen.

Man dürfte wohl auch die Schädigung der Wurzel des Lupinuskeimlinges im destillierten Wasser gleicherweise als Selbstvergiftung auffassen, und dies ist um so wahrscheinlicher, als die Säureausscheidung eine alt bekannte Tatsache ist und die Schädigung durch Erneuerung des Wassers bis zu einem gewissen Grade vermieden werden kann. ${ }^{3)}$ Es kann an diesem Orte aber nicht unsere Aufgabe sein, auf diese Frage näher einzugehen.

\section{Zusammenfassung.}

1. Die stärkereichen Spirogyren im destillierten Wasser scheiden saure Substanzen als Stoffwechselprodukte aus, die schon in kleinen Menge genügen, die Acidität im destillierten Wasser derart zu erhöhen, dass die Spirogyren geschädigt werden. Diese Schädigung der Spirogyren ist also Selbstvergiftung.

2. Deswegen ist es angezeigt, bei gewissen physiologischen Untersuchungen möglichst zu vermeiden, stärkereiche Spirogyren als Versuchsmaterialien zu brauchen.

3. Calciumcarbonat dient als ein ausgezeichnetes Schutzmittel gegen diese Selbstvergiftung der Spirogyren im destillierten Wasser.

1) Es ist auch von Interesse zu sehen, daß die Spirogyren vor diesen Infektionskrankheit desto besser geschützt werden, je größer die Menge von zugesetzten $\mathrm{CaCO}_{3}$ ist.

2) Henderson ('08, '13).

3) Hibbard ('15). 


\section{Sapporo, Botanisches Institute der Universität, im October 1922.}

Nachschrift: Nachdem mein Manuskript ausgeschrieben wurde, habe ich aus dem Buch von CzapeK „Biochemie der Pflanzen“ Bd. II. 1920 (s. 366) ersehen, dass MoLIsch auf die Vorteile ganz schwach alkalischer. Reaktion der Kulturflüssigkeit für die von ihm studierten Algenformen, sowohl Cyanophyceen als höhere Algen, hingewiesen hat. Betreffs der Reaktion der Kulturflüssigkeit stimmt mein Resultat mit dieser schon von Molisch geäusserten Meinung überein. Noch von Interesse ist zu bemerken, dass Calciumcarbonat auch von ihm als einer des geeigneten Mittel empfohlen worden ist, um die passende Acidität der Kulturflüssigkeit zu erreichen.

\section{Literatur-verzeichnis.}

CzapeK, F. (1911). Über eine Methode zur direkten Bestimmung der Oberflächenspannung der Plasmahaut von Pflanzen. Jena.

FUJI, K. (1920). On the conception of "id" and the question of its transmutability (Japanisch). Bot., Mag., Tokyo. vol. 34.

(1921). 細胞ノ構造二關スル定式ノ改定 (Japanisch). Bot. Mag., Tokyo, vol. 35.

Henderson, L. J. (1908). Das Gleichgewicht zwischen Basen und Säuren im tierischen Organismus. Ergeb. Physiol. Bd. 8.

- (1913). The fitness of the environment.

HibBard, R. P. (1915). The question of the toxicity of distilled water. Amer. Journ. Bot. vol. 2.

Hoyt, W. D. (1913). Some toxic and antitoxic effects in cultures of Spirogyra. Bull. Torrey Bot. Club. vol. 40.

Livingston, B. E. (1907). Further studies on the properties of unproductive soils. U. S. Dep't. Agr., Bur. Soils Bull. No. 36.

Loes, J. (1899-1900). On ion-proteid compounds and their role in the mechanics of life phenomena. I. The poisonous character of a pure $\mathrm{NaCl}$ solution. Amer. Journ. Physiol. vol. 3.

- (1903). The relative toxicity of distilled water, sugar solutions and solutions of various constituents of the sea water for marine animals. Univ. Calif. Publ. I.

- (1909). Bioch. Zeitschr. Bd. 15, 16 und 23. zit. nach CzapeK ('11).

Michaelis, L. (1914). Die Wasserstoffionenkonzentration. Berlin.

- (1921). Prakticum der physikalischen Chemie insbesondere der Kolloidchemie für Mediziner und Biologen. Berlin.

NäGELI, C. voN (1893). Über oligodynamische Erscheinung in lebenden Zellen. Denkschr. Schweiz. Naturforsch. Gesell. 33. zit. nach Livingston ('07).

Nakavo, H. (1917). Untersuchungen über die Entwicklungs- und Ernährungsphysiologie einiger Chlorophyceen. Journ. of College of Science, Imper. Univ. Tokyo. vol. 40.

OsTeRHoUt, W. J. V. (1913). Protoplasmic contraction resembling plasmolysis which are caused by pure distilled water. Bot. Mag. vol. 55.

PFEFFER, W. (1904). Pflanzenphysiologie. Bd. II. Leipzig.

True, R. H. (1914). The harmful action of distilled water. Amer. Journ. Bot. vol. 1 . 